\section{Starch granule analysis reveals the functions of stone tools and implications on subsistence economy in Neolithic Yangtze River Basin, China}

\author{
Zhiwei $\mathrm{Wan}^{1}$, Siping $\mathrm{Lin}^{2}$, Min $\mathrm{Ju}^{2}$, \\ Fuqiang Liao ${ }^{2, *}$, Guangming $\mathrm{Zhou}^{3}$ and \\ Yulian Jia ${ }^{2,4}$ \\ ${ }^{1}$ School of Geography and Environmental Engineering, \\ Gannan Normal University, Ganzhou 341000, China \\ ${ }^{2}$ Key Laboratory of Poyang Lake Wetland and Watershed Research \\ Ministry of Education, School of Geography and Environment, \\ Jiangxi Normal University, Nanchang 330022, China \\ ${ }^{3}$ Cultural Relics and Archaeology Institute of Jiangxi Province, \\ Nanchang 330008, China \\ ${ }^{4}$ School of History and Geography, Minnan Normal University, \\ Zhangzhou 363000, China
}

Stone knife and stone muller are common artefacts belonging to the Neolithic period sites in the Yangtze River Basin, China. The Dawangling archaeological site is an important Neolithic site located on the southern bank of the Yangtze River. One stone knife and one stone muller were excavated from the Neolithic layer and examined using starch residue analysis to determine the functions of these tools. More than 165 starch granules and a few bordered pits of tracheid were retrieved from the residues of these stone tools. The results of starch granule analysis show that stone knife and stone muller were probably agricultural processing tools for ancient humans at the Dawangling site during the Neolithic period and hint at the possible existence of a broadspectrum subsistence economy during the Neolithic period.

Keywords: Archaeological sites, starch granule residues, stone tools, subsistence economy, underground storage organs.

CHINA is a global agricultural centre ${ }^{1}$. Many crops have been domesticated in this region, such as common millet (Panicum miliaceum), foxtail millet (Setaria italica) and rice (Oryza sativa) ${ }^{2}$. With regard to subsistence pattern of Neolithic East Asia, the pattern at the Yellow River Basin, China, involves dry farming and is mainly dependent on millets. The pattern at the Yangtze River Basin involves rice farming and is mainly dependent on rice ${ }^{3}$. Nonetheless, many questions arise regarding food utilization at the Yangtze River Basin prior to rice ${ }^{4}$. Researchers suggest the existence of an epoch during which root and tuber crops such as taro and yam were mainly grown in tropical South China before rice cultivation was introduced to this region ${ }^{5}$. Nonetheless, only a few studies have been conducted on the food utilization pattern south of the Yangtze River Basin, thus, further research is necessary.

\footnotetext{
*For correspondence. (e-mail: liaofuqiang@jxnu.edu.cn)
}

With the development of agriculture during the Neolithic period in the Yangtze River Basin in South China, various stone tools began to appear in the archaeological sites. The analysis of these agricultural tools can help us to further understand the agricultural production situation at that time, especially the microremains on the tools can provide direct evidence to determine the functions of these tools. At the same time, by extracting the remains on the surface of these tools, it is possible to gain knowledge on the subsistence economy at that time and reconstruct the pattern of plant utilization by ancient humans in the Neolithic period.

Although the subsistence pattern in the Yellow River Basin and the lower Yangtze River has been widely studied in recent years and clear conclusions have been drawn, few studies focus on the pattern south of the Middle Yangtze River Basin ${ }^{6,7}$. The main reason for this is the lack of necessary research materials and technical methods to determine the macroremains in humid tropic environments ${ }^{8}$. For example, plant macroremains are not preserved in the Latosol, which is widespread in South China $^{5}$. Such macroremains are preserved in the Yellow River Basin, because the alkaline loess is suitable for organic matter preservation. Underground parts of plant organisms (known as underground storage organs, or USOs) can be preserved under carbonization. However, we cannot identify the species of these parts because the remains are too small to discern the overall appearance of tubers and roots. For example, recent developments in palaeoethnobotany and the use of floatation in Chinese archaeological sites have facilitated the detection of broken carbonized roots and tubers in the Zenpiyan site $(12,000-7,000 \mathrm{BP})^{5,9}$. However, these plants cannot be identified due to poor preservation. Thus, we cannot infer food utilization effectively based on the limited amount of macroremains in this region, although a few root or tuber plant macroremains from the Neolithic period have been found in South China.

Pollen grains and phytoliths have been successfully studied in the context of palaeoethnobotany in the past $^{10,11}$. However, root and tuber crops have produced little pollen and hardly any phytoliths ${ }^{12}$. Therefore, we must introduce new plant microfossil research methods and innovative ideas to address the prehistoric palaeoethnobotany problems in South China, especially the Yangtze River Basin. Starch granule analysis technology has recently become an effective archaeobotanical method following phytolith and pollen analyses ${ }^{13-16}$. The basic principle of this analysis involves identifying the microremains recovered from sediments, archaeological artefacts, or even the calculus on human teeth in comparison with a reference collection of modern starch granules. Since the 1980s, the use of starch granule analysis to determine the origins and dispersals of agricultural crops, the functions of stone tools, and palaeoenvironment reconstruction has progressed significantly ${ }^{17,18}$. Previous 
studies have demonstrated that starch granules can be preserved in various environmental contexts for a long period of time ${ }^{19}$.

Thus, we used starch granule analysis to examine the residues on artefact surfaces to determine the functions of stone tools, and use this to acquire information on the subsistence economy at that time. The Dawangling site, Jiujiang County is located $\sim 15 \mathrm{~km}$ south from the Yangtze River and is an important Neolithic site in the Jiangxi Province of China. Thus, this site was selected as the present study region for reconstructing the subsistence pattern of the area south of Yangtze River Basin. No plant macroremains were found in this site during the excavation. Therefore, a new microfossil method can be applied in this area. Here we report the preliminary results obtained for the starch granule analysis of one stone muller and one stone knife excavated from the Dawangling site.

The Dawangling Neolithic archaeological site $\left(29^{\circ} 36^{\prime} 34^{\prime \prime} \mathrm{N}, 115^{\circ} 54^{\prime} 07^{\prime \prime} \mathrm{E}\right)$ is located $\sim 15 \mathrm{~km}$ south of the Yangtze River in Jiujiang County, Jiangxi Province, China (Figure 1). The site is approximately $45 \mathrm{~m}$ amsl and has been excavated from August to October 1976. An archaeological surface measuring $450 \mathrm{~m}^{2}$ was uncovered; this surface was divided into 18 test pits of dimension $5 \mathrm{~m} \times 5 \mathrm{~m}$. The cultural deposits were $1.8-1.9 \mathrm{~m}$ thick and composed of three layers. The first layer consisted of modern cultivated soil, and only a small amount of stone tools was unearthed from the second layer. Thus, most of the stone tools were obtained from the third layer, which was divided into two sub-layers labelled $3 \mathrm{~A}$ and $3 \mathrm{~B}$ based on the sediment characteristics of the site section. All three layers belong to the middle Neolithic strata.

On the basis of their characteristics, archaeologists have confirmed that the artefacts excavated from the

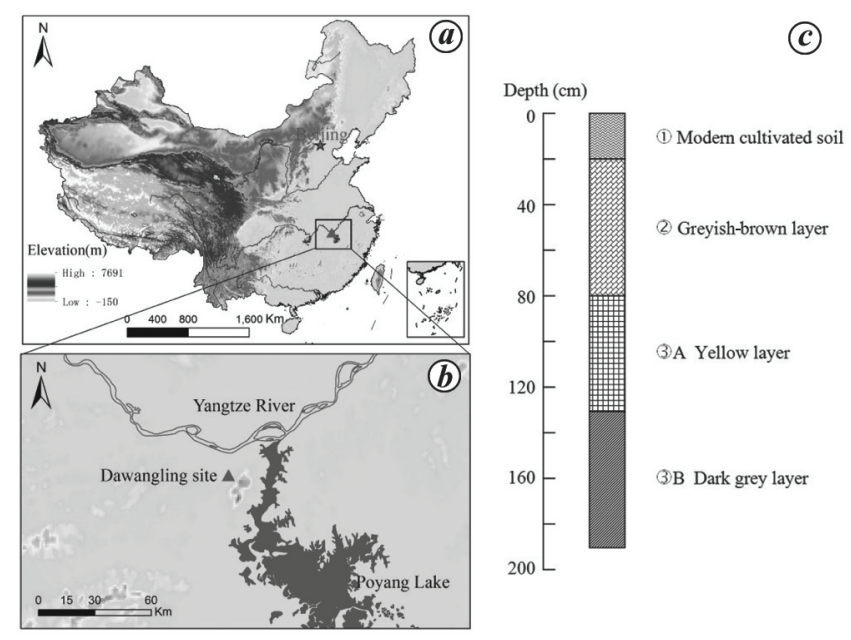

Figure 1. Location of Dawangling site, Jiangxi Province and profile of Dawangling site, China. $\boldsymbol{a}$, The location of the study area in China; $\boldsymbol{b}$, Distribution map of Dawangling site; $\boldsymbol{c}$, Stratigraphic section of the Dawangling site.
Dawangling site belong to the cultural stage between the Zhengjiaao culture (5500 BP) and the late Fanchengdui culture $(\sim 4000 \mathrm{BP})$. Both cultures resided in central Jiangxi. The cultural period of the Dawangling Neolithic site is confirmed to span 5500-5000 BP. The Dawangling site is an important Neolithic site in the Yangtze River Basin, given the scarcity of Neolithic archaeological sites in the northern part of Jiangxi Province and on the south bank of the Yangtze River, which is considered as a pathway through which the northern culture travelled prior to its introduction into the Jiangxi region.

At present, the study area receives approximately $1400 \mathrm{~mm}$ of annual precipitation and approximately $1600 \mathrm{~h} / \mathrm{yr}$ of sunshine. The mean annual temperature at this site is $16^{\circ} \mathrm{C}-17^{\circ} \mathrm{C}$. The vegetation in the region is subtropical evergreen forest type, and the primary crops are rice (O. sativa), oilseed rape (Brassica napus) and cotton (Gossypium hirsutum $)^{20}$.

One stone knife and one stone muller retrieved from the Neolithic strata $3 \mathrm{~A}$ and $3 \mathrm{~B}$ respectively, between August and October 1976, were examined (Figure 2). After excavation, the stone tools were cleaned and placed in clean bags. When the excavation of the Dawangling site was completed, all samples were stored in wooden boxes in the storeroom of the Cultural Relics and Archaeology Institute of Jiangxi Province. Table 1 provides information about these samples. The stone knife, which is made of a type of grey sandstone, is currently fragmentary. The stone muller is almost intact and the crosssection is nearly circular. The surface of the muller was pitted. We also collected some dust in the storeroom as a control sample to detect potential contamination. The dust samples were collected from inside the wooden boxes using a clean brush.

Several areas of each stone tool were sampled to maximize the information obtained on their utilization by the ancient people (Figure 2 and Table 1). The following protocol was used for the extraction of starch granule residues on stone tools: Step 1: The selected area of the stone tools was placed in an ultrasonic water bath and shaken for $10 \mathrm{~min}$. In general, for the end of a small stone knife or stone muller, the sampling process is to place the selected area inside a beaker in an ultrasonic tank so that the water surface in the beaker just touches the surface of the stone tool. For the central part, a larger stone muller, is placed in the beaker and an ultrasonic toothbrush is used for cleaning around the selected area. Step 2: The solution was transferred to a test tube, and $\mathrm{CsCl}$ solution was added at a density of $1.8 \mathrm{~g} / \mathrm{cm}^{3}$ to isolate the starch granules. Step 3: The retrieved residues were mounted on $20 \%$ glycerine and $80 \%$ pure water on a glass slide. The residues were then scanned under polarized and unpolarized light at 400× magnification (using a Nikon eclipse $50 \mathrm{i}$ pol microscope and Mshot digital imaging system (v1.2)). The results were recorded and compared with a modern reference collection. 
Table 1. Information on stone tools and sample data

\begin{tabular}{lccccc}
\hline Tool no. & Type & Length $(\mathrm{cm})$ & Width $(\mathrm{cm})$ & Lab sample no. & Description \\
\hline T8(3)A & Stone knife & 9.5 & 8.5 & $\mathrm{~S} 1$ & Blade section \\
& & & $\mathrm{S} 2$ & Blade section \\
& & & $\mathrm{S} 3$ & Fracture parts \\
& & & $\mathrm{S} 4$ & Stone knife back \\
JD(3)B & Stone muller & 20.1 & 4.5 & $\mathrm{~S} 5$ & Used facet \\
& & & $\mathrm{S} 6$ & Used facet \\
& & & $\mathrm{S} 7$ & Used facet \\
& & & $\mathrm{S} 8$ & Used facet \\
KF1 & & & $\mathrm{S} 9$ & Top parts/used facet \\
\hline
\end{tabular}

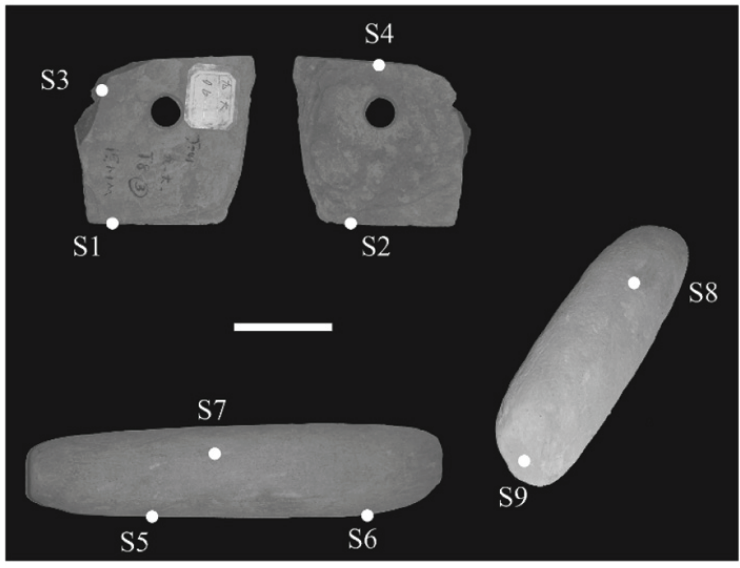

Figure 2. Stone knife (top) and stone muller (bottom) from the Dawangling archaeological site. White dots indicate sampling locations. Scale bar: $5 \mathrm{~cm}$.

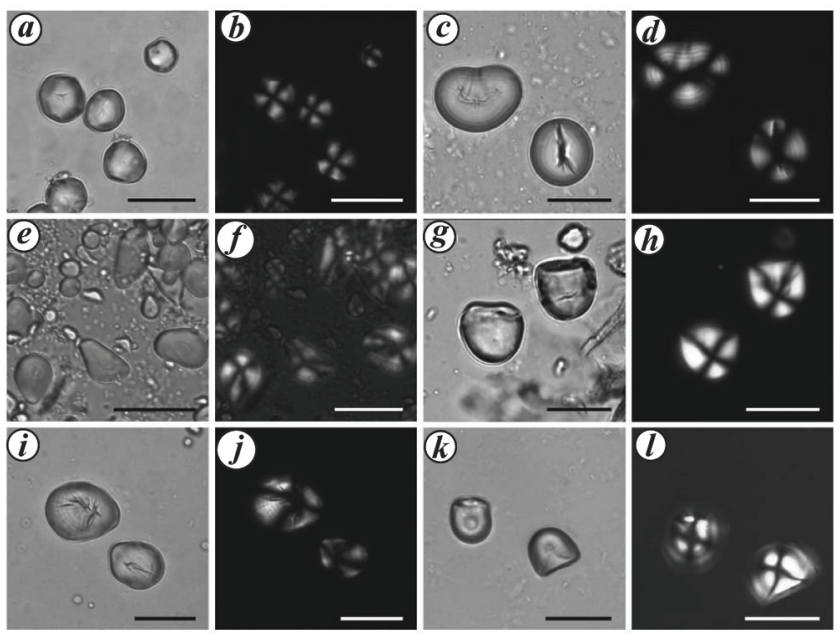

Figure 3. Starch granule reference material derived from modern species in Jiangxi Province for comparison: $\boldsymbol{a}, \boldsymbol{b}$, From Coix chinensis $\boldsymbol{c}, \boldsymbol{d}$, from Vigna angularis; $, \boldsymbol{e}, \boldsymbol{f}$, from Castanea mollissima; $\boldsymbol{g}, \boldsymbol{h}$, from Nymphaea tetragona $; \boldsymbol{i}, \boldsymbol{j}$, from Sagittaria trifolia; $\boldsymbol{k}, \boldsymbol{l}$, from Trachycarpus fortunei. Scale bar: $20 \mu \mathrm{m}$.

To prevent sample contamination, all the laboratory equipment used to extract the starch granules were heated in boiling water and shaken in an ultrasonic water bath before each application.
To identify the starch granules extracted from the stone tools, we compiled more than 50 species from 20 families of plants common in China, particularly in the Jiangxi Province, as modern references (Figure 3). The collected genera include cereals such as Triticum, Panicum, Setaria, Oryza and Coix; acorns such as Quercus and Castanea; roots and tubers such as Lilium, Nelumbo, Dioscorea and Sagittaria; beans such as Vigna and Phaseolus and the stem pith of trees such as Cycas and Trachycarpus. We also consulted descriptions in many other studies of starch granule morphology to categorize the starch granules correctly ${ }^{12,21-24}$.

Samples S1-S9 yielded 165 starch granules that could be classified into seven types, as well as several bordered pits of tracheid (Figure 4). Table 2 shows in detail the distribution of starch granules found in each sample. Starch granules were not obtained from the dust sample KF1.

Type A: This group included 42 polygon starch granules with centric hilum and no visible lamellae. Most starch granules of this type have smooth surface with size ranging from 12.3 to $14.6 \mu \mathrm{m}$, with the exception of seven starch granules. The size of these granules exceeded $15 \mu \mathrm{m}$ and reached up to $19.3 \mu \mathrm{m}$. The modern reference collection suggested that the starch granules derived from panicoid grasses (Poaceae) such as S. italica, P. miliaceum, Sorghum bicolor, Zea mays and Coix lacryma-jobi are almost polygon-shaped ${ }^{25}$. The mean diameter of the starch granules obtained from $S$. italica and $P$. miliaceum was less than $10 \mu \mathrm{m}$. Z. mays originated from America and was brought to the Jiangxi region at a later period. Starch granules from $S$. bicolor always display visible lamellae that are not present in this type ${ }^{24}$, according to the modern references and previous studies.

Although the stone tools came from samples in the storeroom, we sampled and analysed the dust samples in the preservation environment to eliminate possible contamination during preservation. In the course of this experiment, starch granules were analysed simultaneously on the used and non-used facet of all stone tools. According to some studies ${ }^{26-30}$, if the archaeological artefacts are contaminated, the probability of finding starch granules on the used and non-used facet should be the same pattern. 
RESEARCH COMMUNICATIONS

Table 2. Starch granules retrieved from samples S1-S9 at Dawangling site

\begin{tabular}{lcccccccc}
\hline Sample no. & Type A & Type B & Type C & Type D & Type E & Type F & Type G & Percentage \\
\hline S1 & 6 & 1 & 3 & 9 & 1 & 1 & 2 & 42.5 \\
S2 & 11 & 0 & 0 & 5 & 2 & 2 & 3 & 42.5 \\
S3 & 0 & 0 & 1 & 0 & 0 & 0 & 1 & 4 \\
S4 & 0 & 0 & 0 & 2 & 1 & 1 & 2 & 11 \\
S5 & 9 & 7 & 3 & 2 & 2 & 2 & 1 & 23 \\
S6 & 4 & 2 & 7 & 12 & 3 & 1 & 3 & 29 \\
S7 & 8 & 11 & 2 & 9 & 3 & 1 & 0 & 30 \\
S8 & 1 & 0 & 1 & 4 & 0 & 2 & 0 & 7 \\
S9 & 3 & 1 & 2 & 3 & 1 & 0 & 1 & 11 \\
\hline
\end{tabular}

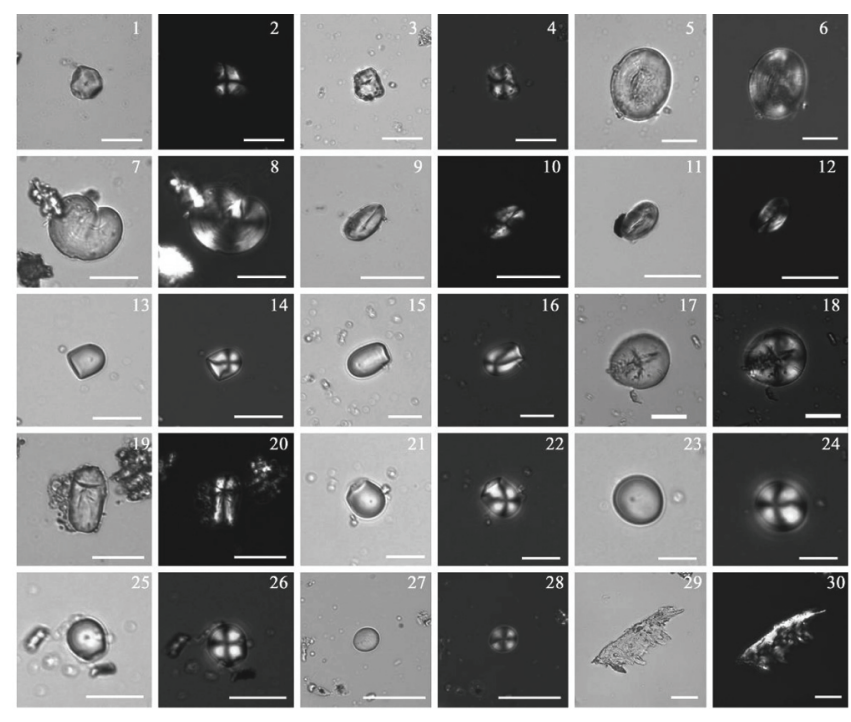

Figure 4. Representative starch granules and bordered pits of tracheid retrieved from stone tools. Each granule is depicted in unpolarized and polarized light views. Type A: $1-4$; type B: $5-8$; type C: 9-12; type D: 13-16; type E: 17-20; type F: 21-26; type G: 27-28; bordered pits of tracheid: 29-30. Scale bar: $20 \mu \mathrm{m}$.

The results of this experiment show that there is a significant difference in the probability of the ancient starch granules extracted from different places of the two stone tools, and the occurrence probability of starch granules on the used facet is significantly higher than that of the non-used facet. On the other hand, $1 \mathrm{~g}$ of corn contains up to 1.7 billion starch grains ${ }^{31-33}$, but due to the natural weathering process of ancient starch grains, only a small amount of starch grains in the crevices of stone tools can be preserved. On the contrary, if there is contamination of modern starch granules, the number of starch granules will be very large. Consequently, some type A granules can be regarded as having originated from Coix spp.

Type B: This group contained 22 elliptic starch granules that usually exhibited a radiating fissure with obvious lamellae. The lamellae were especially clear under polarized light. The size of these starch granules ranged from 19.4 to $46.3 \mu \mathrm{m}$. According to our modern starch granule database and the published literature ${ }^{34}$, the characteristics of this granule type are identical to those ori- ginating from the seeds of leguminous plants (Fabaceae), such as Vigna angularis, Phaseolus vulgaris and Lablab purpureus. Thus, the features of type B are in accordance with those from a type of legume.

Type C: This group contained 19 lenticular and ovalshaped starch granules that were characterized by a curved Maltese extinction cross and a distinctive longitudinal fissure across the hilum. The size of type $\mathrm{C}$ granules ranged from 7.1 to $16.9 \mu \mathrm{m}$, overlapping with the starch granule assemblage from acorn and oak (Fagaceae) ${ }^{35}$. Consequently, the morphological features of type $\mathrm{C}$ are identical to those of acorn and oak, thereby suggesting that this granule type may be derived from a Quercus spp.

Type D: This group contained 46 starch granules with eccentric hilum. Single granules may have been separated from the compound starch granules, which join two or more individual granules. The size of type $\mathrm{D}$ granules ranged from 8.7 to $22.1 \mu \mathrm{m}$. According to the modern reference database, this type of starch granule assemblage may originate from Nelumbo nucifera, Nymphaea tetragona or Schizocapsa plantaginea, which are characterized by semicircles and long ellipses ${ }^{36}$. Although these granules cannot be identified at the species level now, they can be identified to originate from some food like lotus root.

Type E: This group contained 13 ellipsoid and oblong starch granules with eccentric and open hila, and radiating fissures. Some granules showed demonstrable lamellae. The size of these granules ranged from 17.2 to $42.3 \mu \mathrm{m}$. The characteristics of some granules of this type are consistent with those of the Chinese arrowhead (Sagittaria trifolia) in the modern starch granule database ${ }^{24,37}$. The features of the remaining granules of this type are in accordance with the morphological features of some types of roots and tubers, with extreme eccentric hila and curved extinction crosses under polarized view. Accordingly, type E may have been derived from some species of Sagittaria or other USOs.

Type F: This group contained ten elliptic or almost round starch granules with eccentric, open hila and unclear lamellae under unpolarized light. Their size ranged from 15.4 to $26.8 \mu \mathrm{m}$. Based on previous studies, these granules may originate from some species of Arecaceae 
(palms). A comparison with the modern references indicated that the characteristics of type $\mathrm{F}$ are similar to those of Caryota urens, Areca catechu and starch granules from the pitch of some types of palm ${ }^{37}$. Consequently, this granule type can be considered to have originated from the stem pith of palms.

Type G: This group contained 11 small, circular, starch granules with no fissure on the surface and closed hila. The granule size ranged from 3.7 to $6.9 \mu \mathrm{m}$. Two starch granules ranging in size from 21.2 to $23.5 \mu \mathrm{m}$ may be damaged. Distinctive features were lacking, and many plants contained starch granules resembling a small sphere. Thus, the starch granules in this group are not identifiable at present, and we regard this type as unidentified.

Bordered pits of tracheid: In the course of this experiment, several bordered pits of tracheid were recovered from the residues extracted from the stone knife sample. Previous studies have demonstrated that bordered pits of tracheid are common in conifers ${ }^{38,39}$. These pits can exhibit an extinction cross under polarized light, similar to starch granules under polarized light. Thus, it may confuse these pits with compound starch granules, when the observation is not careful.

Several starch granules were recovered from the stone knife and the stone muller. For the stone knife, almost $85 \%$ of the total starch granule assemblage was retrieved from samples S1 and S2, which come from the knife blade and thus likely reflect its use. Since the extracted starch granule residues mainly come from the used facet of stone tools, this can reflect ancient plant utilization information. Only $11 \%$ and $4 \%$ of the granules were obtained from the back portion of the knife and the fractured parts respectively. This distribution of starch residues indicates that the stone knife had not been contaminated by secondary deposition of the stratum environment ${ }^{40}$. It is generally considered that if the stone tools are contaminated, the probability of starch granules being extracted from all parts of the tools is the same, and the number of starch granules extracted will far exceed the number of ancient starch granule microremains ${ }^{27,28,30}$. Moreover, no starch granule was detected on the dust sample from the storeroom, thereby suggesting that the batch of archaeological items stored in the room after excavation had not been contaminated during storage. Starch granules were evenly distributed across the stone muller. This phenomenon may indicate that the muller was a multipurpose tool. For example, the muller can be used to grind plants, and thereby cause the starch granule residues at the S5-S8 sampling positions to be consistent. This tool can also pound food materials as a pestle; hence, S9 makes contact with plants as such.

Experiments have also demonstrated that starch granules can be enlarged in the process of grinding and pounding ${ }^{41}$. External forces influence these granules and alter them morphologically. Such forces also cause the extinction cross to weaken under polarized light. Figure 5 indicates that the interior structure of lamellae can be observed clearly under unpolarized light. The left part of the starch granule shown in Figure $5 c$ may have experienced a strong external punching force; thus, a depressed region was observed on the surface. The left part in Figure $5 d$ lost the extinction cross property and the border of the granule is marked by an arrowhead.

Various starch granules were identified in this study and we can use the information to infer the uses of stone knife and stone muller based on the results of the microremains of starch granules. The starch granules from Dawangling may have been derived from Coix spp., food legumes, Quercus spp., lotus root, a unidentified USO and palms. Figure 6 shows the percentage of each type of starch granule. The radar diagram indicates that the main edible plants processed by the stone knife and stone muller were Coix spp. and lotus root. According to the local modern vegetation type and ethnobotanical records, lotus, USO and Arecaceae are common plant resources in the tropical zones ${ }^{5,42}$. For instance, N. tetragona originated from tropical India. Cassava remains an important staple food resource in tropical America, and indigenous people residing in southeastern Asia still eat sago made from the starch of Arecaceae pith ${ }^{12}$. Quercus spp. (oak) is currently a common plant in northern China. The Dawangling site is located in South China, which is a rice-farming region. Consequently, the food utilization revealed by starch residues also contained some kind of non-staple food.

Unlike previous studies that labelled the people who lived in the Yangtze River region as mainly rice farmers ${ }^{5}$, those who lived in the Dawangling region during the Neolithic period may have relied on non-staple foods,
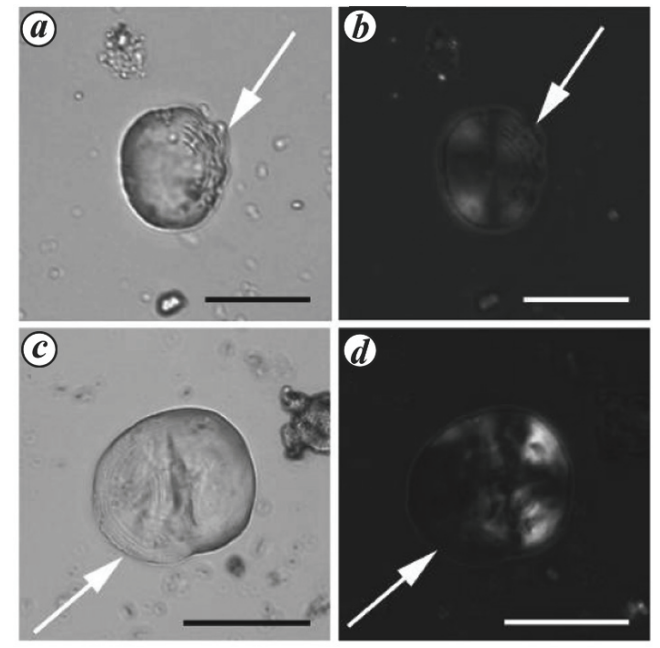

Figure 5. Damaged starch granules retrieved from samples S5 and S9 of the stone muller from Dawangling site. $\boldsymbol{a}$, Interior structure of lamellae; $\boldsymbol{b}$, Interior structure of lamellae under polarized light; $\boldsymbol{c}$, Starch granule experienced external punching force; $\boldsymbol{d}$, Starch granule experienced external punching force under polarized light. White arrowheads indicate damaged parts of the starch granules. Scale bar: $20 \mu \mathrm{m}$. 


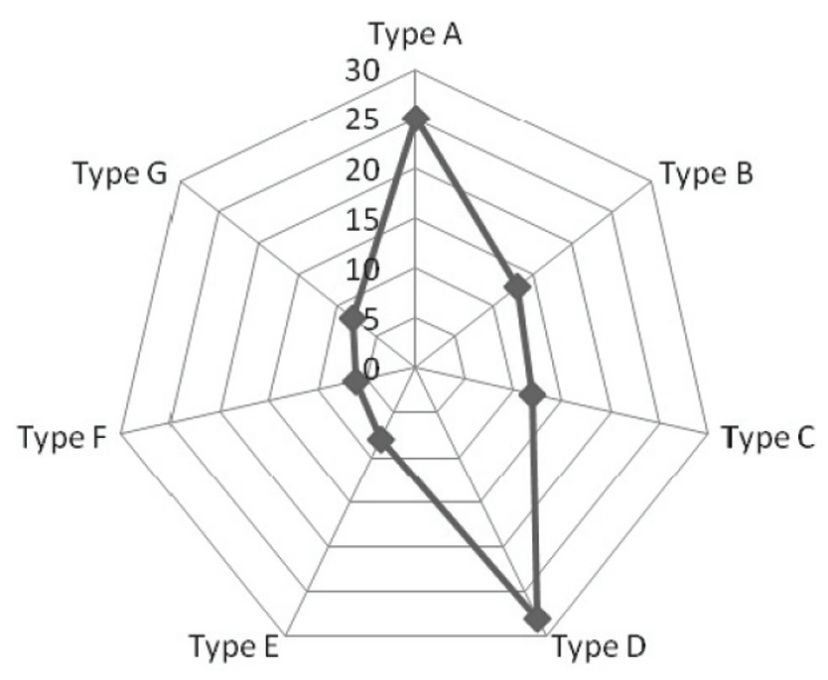

Figure 6. Radar diagram of the percentage of each type of starch granule.

including cereals, beans, oak, lotus, USO and palms. These results of this study suggest that until the midNeolithic age, the gathering economy remained important to the ancient people. Moreover, the rice farming system may be have been adopted later as the main food resource. No rice starch granule was detected in this study, probably because of the tendency of rice starch granules to weather given their small size ${ }^{38}$. Nonetheless, this phenomenon does not affect the use of the starch assemblage information to reconstruct food utilization pattern.

A certain number of different types of plant starch granules were extracted in this experiment, reflecting the diversity of plant utilization by ancient humans in the study area. Approximately half of these starch granules originated from underground storage organs, which had not been deciphered earlier.

The starch granule analysis results show that the stone knife and stone muller unearthed from the Dawangling Neolithic site have complicated agricultural production functions. The stone knife can be used to harvest and scrape crops, and the stone muller can process and grind starchy plants. The microremains of starch granules also indicate that before adopting rice farming, ancient humans at the Dawangling site also used many plant food resources such as Coix spp. with those of beans and oaks.

Conflicts of interest: The authors declare no conflict of interest.

1. Larson, G. et al., Current perspectives and the future of domestication studies. Proc. Natl. Acad. Sci. USA, 2014, 111(17), 61396146.

2. Zhao, Z., The Middle Yangtze region in China is one place where rice was domesticated: phytolith evidence from the Diaotonghuan Cave, northern Jiangxi. Antiquity, 1998, 72, 885-897.
3. Fuller, D. Q., Harvey, E. and Qin, L., Presumed domestication? Evidence for wild rice cultivation and domestication in the fifth millennium BC of the Lower Yangtze region. Antiquity, 2007, 81(312), 316-331.

4. Yang, X. and Perry, L., Identification of ancient starch grains from the tribe Triticeae in the North China Plain. J. Archaeol. Sci., 2013, 40(8), 3170-3177.

5. Zhao, Z., Paleoethnobotany: Theories, Methods and Practice, Science Press, Beijing, China, 2010.

6. Lu, H. Y. et al., Earliest domestication of common millet (Panicum miliaceum) in East Asia extended to 10,000 years ago. Proc. Natl. Acad. Sci. USA, 2009, 106(18), 7367-7372.

7. Jiang, L. and Liu, L., New evidence for the origins of sedentism and rice domestication in the Lower Yangzi River, China. Antiquity, 2006, 80(308), 355-361.

8. Piperno, D. R., Ranere, A. J., Holst, I. and Hansell, P., Starch grains reveal early root crop horticulture in the Panamanian tropical forest. Nature, 2000, 407(6806), 894-897.

9. Messner, T. C. and Schindler, B., Plant processing strategies and their affect upon starch grain survival when rendering Peltandra virginica (L.) Kunth, Araceae edible. J. Archaeol. Sci., 2010, 37(2), 328-336.

10. Zarrillo, S., Pearsall, D. M., Raymond, J. S., Tisdale, M. A. and Quon, D. J., Directly dated starch residues document early formative maize (Zea mays L.) in tropical Ecuador. Proc. Natl. Acad. Sci. USA, 2008, 105(13), 5006-5011.

11. Piperno, D. R. and Jones, J. G., Paleoecological and archaeological implications of a Late Pleistocene/Early Holocene record of vegetation and climate from the Pacific coastal plain of Panama. Quaternary Res., 2003, 59(1), 79-87.

12. Torrence, R. and Barton, H., Ancient Starch Research, Left Coast Press, Walnut Creek, USA, 2006.

13. Henry, A. G., Hudson, H. F. and Piperno, D. R., Changes in starch grain morphologies from cooking. J. Archaeol. Sci., 2009, 36(3), 915-922.

14. Duncan, N. A., Pearsall, D. M. and Benfer, R. A., Gourd and squash artifacts yield starch grains of feasting foods from preceramic Peru. Proc. Natl. Acad. Sci. USA, 2009, 106(32), 13202 13206.

15. Holst, I., Moreno, J. E. and Piperno, D. R., Identification of teosinte, maize, and Tripsacum in Mesoamerica by using pollen, starch grains, and phytoliths. Proc. Natl. Acad. Sci. USA, 2007, 104(45), 17608-17613.

16. Guan, Y., Pearsall, D. M., Gao, X., Chen, F., Pei, S. and Zhou, Z., Plant use activities during the Upper Paleolithic in East Eurasia: evidence from the Shuidonggou site, Northwest China. Quaternary Int., 2014, 347(1), 74-83.

17. Perry, L. et al., Starch fossils and the domestication and dispersal of chili peppers (Capsicum spp. L.) in the Americas. Science, 2007, 315(5814), 986-988.

18. Perry, L., Starch analyses reveal the relationship between tool type and function: an example from the Orinoco valley of Venezuela. J. Archaeol. Sci., 2004, 31(8), 1069-1081.

19. Mercader, J., Bennett, T. and Raja, M., Middle Stone Age starch acquisition in the Niassa Rift, Mozambique. Quaternary Res., $2008,70(2), 283-300$.

20. Ren, M., Yang, R. and Bao, H., An Outline of the Physical Geography of China, Foreign Language Press, Beijing, China, 1985, pp. $1-471$.

21. Tao, D., Zhang, J., Zheng, W., Cao, Y., Sun, K. and Jin, S. A., Starch grain analysis of human dental calculus to investigate Neolithic consumption of plants in the middle Yellow River Valley, China: a case study on Gouwan site. J. Archaeol. Sci. Rep., 2015, 2, 485-491.

22. Gong, Y. et al., Investigation of ancient noodles, cakes, and millet at the Subeixi Site, Xinjiang, China. J. Archaeol. Sci., 2011, 38(2), $470-479$. 
23. Yang, X., Ancient starch research in China: progress and problems. Quaternary Sci., 2017, 37(1), 196-210.

24. Wan, Z. W., Yang, X. Y., Ge, Q. S., Fan, C. S., Zhou, G. M. and Jiang, M. X., Starch grain analysis reveals Late Neolithic plant utilization in the middle reaches of the Ganjiang River. Sci. China Earth Sci., 2012, 55(12), 2084-2090.

25. Li, M., Yang, X., Wang, H. and Wang, Q., Starch grains from dental calculus reveal ancient plant foodstuffs at Chenqimogou site, Gansu Province. Sci. China (Earth Sci.), 2010, 20(5), 694-699.

26. Ma, Z., Li, Q., Huan, X., Yang, X., Zheng, J. and Ye, M., Plant microremains provide direct evidence for the functions of stone knives from the Lajia site, northwestern China. Chin. Sci. Bull., 2014, 59(11), 1151-1158.

27. García-Granero, J. J., Starch taphonomy, equifinality and the importance of context: some notes on the identification of food processing through starch grain analysis. J. Archaeol. Sci., 2020, 124, 105267.

28. Tao, D., Zhang, G., Zhou, Y. and Zhao, H., Investigating wheat consumption based on multiple evidences: stable isotope analysis on human bone and starch grain analysis on dental calculus of humans from the Laodaojing cemetery, Central Plains, China. Int. J. Osteoarchaeol., 2020, 30(5), 594-606.

29. Wan, Z. W., Lin, S. P., Ju, M., Ling, C. H. and Liao, F. Q., Morphotypological analysis of starch granules through discriminant method and its application in plant archeological samples. Appl. Ecol. Environ. Res., 2020, 18(3), 4595-4608.

30. Berman, M. J. and Pearsall, D. M., Crop dispersal and Lucayan tool use: investigating the creation of transported landscapes in the Central Bahamas through starch grain, phytolith, macrobotanical, and artifact studies. J. Field Archaeol., 2020, 45(5), 355-371.

31. Zhang, L., Starch Sugar, China Light Industry Press, Beijing, China, 1988.

32. BeMiller, J. N. and Whistler, R. L., Starch: Chemistry and Technology, Academic Press, Burlington, USA, 2009.

33. Bertolini, A., Starches: Characterization, Properties, and Applications, CRC Press, Boca Raton, Florida, USA, 2009.

34. Piperno, D. R. and Dillehay, T. D., Starch grains on human teeth reveal early broad crop diet in northern Peru. Proc. Natl. Acad. Sci. USA, 2008, 105(50), 19622-19627.
35. Liu, L., Field, J., Fullagar, R., Zhao, C., Chen, X. and Yu, J., A functional analysis of grinding stones from an early holocene site at Donghulin, North China. J. Archaeol. Sci., 2010, 37(10), 2630 2639.

36. Yang, X. et al., Early millet use in northern China. Proc. Natl. Acad. Sci. USA, 2012, 109(10), 3726-3730.

37. Yang, X. et al., Sago-type palms were an important plant food prior to rice in southern subtropical China. PLOS ONE, 2013, 8(5), e63148.

38. Yang, X. and Jiang, L., Starch grain analysis reveals ancient diet at Kuahuqiao site, Zhejiang Province. Chin. Sci. Bull., 2010, 55(12), 1150-1156.

39. Innes, J. B., Zong, Y., Chen, Z., Chen, C., Wang, Z. and Wang, H., Environmental history, palaeoecology and human activity at the early Neolithic forager/cultivator site at Kuahuqiao, Hangzhou, eastern China. Quaternary Sci. Rev., 2009, 28(23-24), 2277-2294.

40. Barton, H., Torrence, R. and Fullagar, R., Clues to stone tool function re-examined: comparing starch grain frequencies on used and unused obsidian artefacts. J. Archaeol. Sci., 1998, 25(12), 12311238.

41. Liu, L. et al., Plant exploitation of the last foragers at Shizitan in the Middle Yellow River Valley China: evidence from grinding stones. J. Archaeol. Sci., 2011, 38(12), 3524-3532.

42. Flad, R., Li, S. C., Wu, X. H. and Zhao, Z. J., Early wheat in China: Results from new studies at Donghuishan in the Hexi Corridor. Holocene, 2010, 20(6), 955-965.

ACKNOWLEDGEMENT. This study was supported by the National Natural Science Foundation of China (41761045).

Received 13 May 2020; revised accepted 1 February 2021

doi: $10.18520 / \mathrm{cs} / \mathrm{v} 120 / \mathrm{i} 9 / 1520-1526$ 